\title{
Gerenciamento intra-hospitalar dos resíduos químicos perigosos manuseados pela enfermagem
}

\author{
Intra-hospital management of hazardous chemical waste handled by nurses \\ Gestión intrahospitalaria de los residuos químicos peligrosos manejados por la enfermería
}

\author{
Taiza Florencio Costa'; Vanda Elisa Andres Felli"; Maritza Consuelo Ortiz Sanchez"ll; \\ Simone Cruz Machado Ferreiralv; Zenith Rosa Silvinov; Deise Ferreira de Souza ${ }^{v \prime}$
}

\begin{abstract}
RESUMO
Objetivo: avaliar as etapas de gerenciamento dos resíduos químicos perigosos - formaldeído e ortoftaldeído, manuseados pela enfermagem. Método: pesquisa descritiva e coleta de dados realizada de setembro a dezembro de 2012, no Hospital Universitário da Universidade de São Paulo, por meio da aplicação da Ficha de Informação de Gerenciamento de Resíduo Químico Perigoso dos resíduos formaldeído e ortoftaldeído gerados, respectivamente, no centro obstétrico e endoscopia. Projeto aprovado por Comitê de Ética em Pesquisa. Resultados: a análise estatística dos dados evidenciou que foram atendidas 3 (13\%) especificações do total de 23 (100\%) necessárias, com prevalência da identificação, seguida da segregação e nenhuma do transporte interno dos resíduos. Há necessidade de a instituição cumprir as especificações de identificações gerais em relação ao número total de avaliações nos recipientes de resíduos de formaldeído e ortoftaldeído. Conclusão: esta pesquisa proporcionou importantes diretrizes para a elaboração do Plano de Gerenciamento de Resíduos Químicos Perigosos da atenção hospitalar.

Descritores: Enfermagem do trabalho; resíduos químicos; saúde do trabalhador; saúde ambiental.
\end{abstract}

\section{ABSTRACT}

Objective: to evaluate the stages in management of hazardous chemical waste handled by nursing staff. Method: in this descriptive study, data was collected from September to December 2012 at São Paulo University Hospital, by applying the Hazardous Chemical Waste Management Information Sheet for formaldehyde and ortho-phthalaldehyde waste in the obstetric center and endoscopy, respectively. The study was approved by the research ethics committee. Results: data analysis showed that 3 (13\%) of the 23 (100\%) required specifications were met, predominantly identification, followed by segregation, and that none on internal waste transportation were met. The institution needs to comply with general identification specifications regarding the total number of evaluations of formaldehyde and orthophthaldehyde waste containers. Conclusion: this study also yielded important guidelines for preparation of the hospital care Hazardous Chemical Waste Management Plan.

Descriptors: Nursing work; chemical waste; occupational health; environmental health.

\section{RESUMEN}

Objetivo: evaluar las etapas de la gestión de los residuos químicos peligrosos - formaldehído y ortoftaldehído, manejados por la enfermería. Métodos: investigación descriptiva y recolección de datos realizada, de septiembre a diciembre de 2012, en el Hospital Universitario de la Universidad de São Paulo, por medio de la aplicación de la Ficha de Información de Gestión de Residuo Químico Peligroso de los residuos de formaldehído y ortoftaldehído generados, respectivamente, en el Centro de Obstetricia y Endoscopía. Proyecto aprobado por el Comité de Ética en Investigación. Resultados: el análisis estadístico de los datos mostró que fueron atendidas $3(13 \%)$ especificaciones del total de $23(100 \%)$ requeridas, con prevalencia de la identificación, seguida por la segregación y ninguna relacionada al transporte interno de los residuos. Es necesario que la institución cumpla con las especificaciones de identificaciones generales en relación al número total de evaluaciones en los recipientes de residuos de formaldehído y ortoftaldehído. Conclusión: la investigación proporcionó importantes directrices para la elaboración del Plan de Gestión de Residuos Químicos Peligrosos de la atención hospitalaria.

Descriptores: Enfermería del trabajo; residuos químicos; salud ocupacional; salud ambiental.

\section{INTRODUÇÃO}

O uso de produtos químicos na atenção hospitalar traz grandes benefícios à população e, ao mesmo tempo, grandes preocupações em relação à geração dos resíduos químicos perigosos (RQP), uma vez que as falhas no gerenciamento dos mesmos podem acarretar males à saúde humana e ambiental.

O gerenciamento de resíduos químicos perigosos (GRQP) compreende etapas de manejo intra e extra estabe-

Enfermeira. Doutora em Ciências. Professora Adjunta, Universidade Federal Fluminense. Brasil. E-mail: taizaflorencio@hotmail.com "Enfermeira. Doutora em Enfermagem. Professora Sênior, Universidade de São Paulo. Brasil. E-mail: vandaeli@usp.br '"Enfermeira. Doutora em Enfermagem. Professora Adjunta, Universidade Federal Fluminense. Brasil. E-mail: morsa_peru@yahoo.com IVEnfermeira. Doutora em Enfermagem. Professora Associada, Universidade Federal Fluminense. Brasil. E-mail: s.cruz.ferreira@uol.com.br VEnfermeira. Doutora em Enfermagem. Professora Titular, Universidade Federal Fluminense. Brasil. E-mail: zenithrosa52@gmail.com

v'Enfermeira. Doutora em Ciências do Cuidado em Saúde. Professora Adjunta, Universidade Federal Fluminense, Brasil. E-mail: dfsnit@hotmail.com vil Agradecimento à Coordenação de Aperfeiçoamento de Pessoal de Nível Superior, que financiou o estudo: Gerenciamento de recursos humanos em enfermagem no contexto assistencial, ético, político e financeiro de hospitais gerais, públicos, de ensino do município de São Paulo. Universidade de São Paulo, 2014. 
lecimento de saúde. As etapas do intra-hospitalar envolvem a segregação, acondicionamento, identificação, transporte interno e armazenamento nos abrigos de resíduos.

Neste estudo a ênfase compreende da segregação até o transporte interno, uma vez que tais fases são imprescindíveis para não comprometer o restante do processo, inclusive no extra-hospitalar. Nesse contexto, ao avaliar a gestão dos RQP, também se considera que as atividades do enfermeiro podem ou não trazer danos à sua saúde. Assim, requer atenção com a finalidade de reduzir riscos no trabalho ${ }^{1}$.

A Resolução da Diretoria Colegiada (RDC), da Agência Nacional de Vigilância Sanitária (ANVISA), no 306/2004, dispõe sobre o Regulamento Técnico para o Gerenciamento de Resíduos de Serviços de Saúde e estabelece que todo gerador de resíduos de serviço de Saúde (RSS) deverá elaborar um Plano de Gerenciamento de Resíduos de Serviços de Serviços de Saúde (PGRSS) incluindo-se os RQP pertencentes ao grupo de resíduos químicos (RQ) conhecidos Grupo B. O manejo dos RSS é entendido como a ação de gerenciar os resíduos em seus aspectos intra e extra estabelecimento, desde a geração até a disposição final, incluindo as seguintes etapas no intra-hospitalar²: Segregação: é a separação dos resíduos, no momento e local de sua geração; Acondicionamento: consiste no ato de embalar os resíduos segregados; Identificação: é o conjunto de medidas que permite o reconhecimento dos resíduos. Assim, o Grupo B é identificado pelo símbolo de risco associado; Transporte interno: consiste no translado dos resíduos dos pontos de geração até o local destinado ao armazenamento temporário ou externo.

Entre os produtos químicos perigosos consumidos no ambiente hospitalar destacam-se o formaldeído e o ortoftaldeído. O gerenciamento adequado dos resíduos gerados pelos estabelecimentos de saúde é fundamental para a promoção da saúde, qualidade de vida e preservação do meio ambiente ${ }^{3}$. Assim, este estudo objetivou avaliar as etapas de gerenciamento dos RQP - formaldeído e ortoftaldeído, manuseados pela enfermagem.

\section{REVISÃo DE LITERATURA}

O formaldeído, enquanto um conservante de tecidos humanos, desinfetante e esterilizante, ao tornar-se um dos RQP, gerenciado de forma insegura em qualquer uma das fases do manejo, poderá em função do seu princípio ativo, ser absorvido pelas vias oral, dérmica e inalatória, apresentando elevado potencial de irritabilidade das mucosas ocular e do trato respiratório, chegando, inclusive, a ocasionar danos celulares, levando a carcinogenicidade, sendo ainda fator agravante na promoção de males à fauna e flora ${ }^{4,5}$.

O ortoftaldeído é um desinfetante de alto nível, desenvolvido para o reprocessamento de artigos odonto-médico-hospitalares sensíveis ao calor. Tal produto apresenta-se como um substituto do glutaraldeído, sendo um dialdeído com ação comprovada contra micro-organismos vegetativos, vírus da hepatite B e C e, especialmente, contra as microbactérias resistentes ao calor e ao próprio glutaraldeído.

O ortoftaldeído, tanto na forma de produto como de resíduo, pode ocasionar sensibilização cutânea, irritação ocular e respiratória, além de ser prejudicial para o meio ambiente, sendo necessárias precauções no manuseio do produto e resíduo ${ }^{6}$.

Nesse contexto, ao se considerar a relevância do gerenciamento intra-hospitalar dos RQP de formaldeído e de ortoftaldeído manuseados pela Enfermagem, este estudo buscou a avaliação ao atendimento do Regulamento Técnico para o Gerenciamento de Resíduos de Serviços de Saúde por meio das especificações da Ficha de Informação de Gerenciamento de Resíduo Químico Perigoso Hospitalar (FIGERQP-HOSP) ${ }^{7}$, gerada a partir da análise dos dados, da tese Gerenciamento de resíduos químicos perigosos manuseados pela Enfermagem de um hospital universitário ${ }^{7}$ que evidenciou o desconhecimento da Enfermagem quanto ao GRQP, gerados na assistência ao paciente.

Ainda, o mesmo estudo detectou a lacuna existente entre as orientações de uso dos produtos e informações de segurança sobre o que fazer com seus resíduos, fato este observado mesmo para os produtos acompanhados da Ficha de Informações de Segurança de Produtos Químicos (FISPQ).

\section{Metodologia}

Trata-se de um estudo quantitativo, descritivo, uma vez que tem como objetivo primordial avaliar o gerenciamento das etapas de segregação, acondicionamento, identificação e transporte interno de dois tipos dos RQP. A pesquisa foi realizada no Hospital Universitário da Universidade de São Paulo (HU-USP) envolvendo os RQP de formaldeído e ortoftaldeído, gerados, respectivamente, no Centro Obstétrico e Endoscopia. Para coleta de dados foi utilizada a FIGERQP-HOSP ${ }^{7}$, no que se refere ao GRQP no intra-hospitalar. O estudo em questão foi aprovado pelo Comitê de Ética em Pesquisa do HU-USP no 1183/12, sob o Certificado de Apresentação para Apreciação Ética (CAAE) no 0132.0.196.198-11. A coleta nos recipientes dos RQP dispensou a assinatura do Termo de Consentimento Livre e Esclarecido (TCLE). Os dados foram coletados no período de setembro a dezembro de 2012, totalizando 16 avaliações.

$\mathrm{Na}$ operacionalização da coleta de dado, por meio da FIGERQP-HOSP, os resíduos de formaldeído de ortoftaldeído foram avaliados quanto às especificações registradas nos coletores de RQ, armazenados no abrigo externo de resíduos, avaliando-se dados sobre estabilidade; corrosividade; reatividade; toxicidade; informações ambientais; princípio ativo; classificação; 
indicações de segregação; acondicionamento; identificação e transporte interno. Os dados foram sistematizados, inseridos em bancos de dados, submetidos à análise estatística e mostrados em tabelas segundo as frequências absoluta e relativa.

\section{RESULTADOS E DiscusSÃo}

$\mathrm{Na}$ avaliação do sistema de gerenciamento intra-hospitalar dos resíduos de formaldeído e ortoftaldeído, segundo os critérios da FIGERQP-HOSP, evidenciou-se o cumprimento a 3 (13\%) das 23(100\%) especificações necessárias (oito especificações sobre as etapas de segregação e acondicionamento; 11 de identificação geral no coletor e quatro especificações da etapa de transporte interno) para o adequado gerenciamento dos 132 litros de resíduo de ortoftaldeído gerados mensalmente na endoscopia, e 75 litros de resíduos de formaldeído do centro cirúrgico.

Observou-se que nas etapas da segregação e acondicionamento, durante as 16 avaliações, das oito (100\%) especificações nos recipientes dos resíduos, somente $1(12,5 \%)$ atendeu às recomendações de FIGERQP-HOSP, sendo representado pela informação do princípio ativo no rótulo da embalagem dos $R Q$; as demais não foram atendidas, conforme mostra a Tabela 1.

Os dados evidenciaram que a segregação e acondicionamento dos RQP estudados vêm sendo efetuados, o que é um fator positivo, no entanto, ocorrem não conformidades quanto às informações indispensáveis como: sinônimos, composição do resíduo, composição, ingredientes perigosos, características de periculosidade, informações toxicológicas, indicação de não ser misturado com outros resíduos (indicação de segregar separadamente) e a compatibilidade com o recipiente coletor (indicação do tipo de coletor adequado para acondicionamento).

Segundo a norma técnica P4.262, da Companhia Ambiental do Estado de São Paulo (CETESB), a segregação dos RQP deverá ser prevista no momento da geração, conforme a classificação e destinação final dos mesmos.

TABELA 1: Especificações de segregação e acondicionamento em relação ao número total de avaliações nos recipientes de resíduos de formaldeído e ortoftaldeído. Brasil, 2012. (N=16)

\begin{tabular}{lcccc}
\hline $\begin{array}{l}\text { Especificações de segregação e } \\
\text { acondicionamento no coletor }\end{array}$ & $\begin{array}{c}\text { Pre- } \\
\text { sente }\end{array}$ & $\%$ & $\begin{array}{c}\text { Au- } \\
\text { sente }\end{array}$ & $\%$ \\
\hline Princípio ativo & 16 & 100 & - & - \\
Sinônimos & - & - & 16 & 100 \\
Composição & - & - & 16 & 100 \\
$\begin{array}{l}\text { Ingredientes perigosos } \\
\text { Característica de } \\
\text { periculosidade }\end{array}$ & - & - & 16 & 100 \\
$\begin{array}{l}\text { Informações toxicológicas } \\
\text { Indicação de segregar }\end{array}$ & - & - & 16 & 100 \\
$\begin{array}{l}\text { separadamente } \\
\text { Indicação do tipo de coletor } \\
\text { adequado p/acondicionamento }\end{array}$ & - & - & 16 & 100 \\
\hline
\end{tabular}

Assim, a não segregação implicará na destinação final inadequada e, em alguns casos, com mistura de resíduos ${ }^{8}$.

Os resíduos líquidos devem ser acondicionados em recipientes constituídos de material compatível com o resíduo gerado, devendo ainda, atender às características de resistência e rigidez, sendo ainda estanques, e com tampa de rosca vedante ve $^{2,9,10}$.

Os $\mathrm{RQ}$, como o glutaraldeído e outros aldeídos como o ortoftaldeído, devem ser segregados, armazenados em recipientes de plástico rígido, identificados e avaliados quanto à indicação de serem encaminhados à incineração em indústrias químicas com licenciamento ambiental para essa prática ${ }^{11}$.

Estudos indicam que alguns fatores estão associados à ineficiência na etapa da segregação, como a falta de coletores apropriados ao descarte dos resíduos e o treinamento deficiente dos profissionais que lidam com a segregação dos resíduos no local de geração ${ }^{12,13}$.

Existe uma ênfase maior das pesquisas em relação à fase de segregação dos RSS de acordo com as características físicas, químicas, biológicas, riscos envolvidos e seu estado físico, uma vez que as outras etapas dependem da separação do resíduo no momento e local de geração ${ }^{7}$. Apesar da segregação dos resíduos ser a etapa do manejo mais enfatizada nos estudos, as falhas nessa etapa promovem a mistura dos diversos tipos de resíduos com os resíduos infectantes ${ }^{14}$.

Estudos relataram que em muitas instituições de saúde, em diferentes países, não existe a segregação dos resíduos infecciosos, inflamáveis, tóxicos e outros materiais que requerem manuseio especial, além da falta de identificação, dos recipientes, carrinhos de transporte interno e abrigo interno de resíduos ${ }^{15-17}$.

Diante disso, ainda é frequente os estudos abordarem os RQP de forma generalizada entre os resíduos infectantes e perfurocortantes.

Em função dos riscos, os produtos químicos deverão ser segregados como os RQP na ocorrência de: mudança de temperatura, o avencimento, a quebra acidental, a interdição de uso, a identificação ilegível, a perda de lacre, que coloque em risco o produto, a alteração de cor e/ou a presença de corpo estranho ${ }^{7}$.

Por meio deste estudo, verificou-se, ainda que as ocorrências de segregação dos RQP que a instituição envolve, ou seja, basicamente, medicamentos vencidos e/ou contaminados, quimioterápicos, glutaraldeído, formaldeído e xilol; todos, normalmente, manuseados pela enfermagem ${ }^{7}$. A segregação do ortoftalaldeído, enquanto um substituto do glutaraldeído, requer cuidados específicos em função de sua periculosidade.

Pesquisa sobre o Plano de Gerenciamento de Resíduos de Serviços de Saúde em unidades hospitalares da Região Metropolitana da Baixada Santista evidenciou que os resíduos do Grupo B, na sua maioria, foram representados por medicamentos vencidos ou não utilizados ${ }^{18}$. 
Em muitos locais, o que se observa é um comportamento de tudo ou nada: ou todos os resíduos são segregados como perigosos, ou nada é separado ${ }^{19}$.

Quanto ao acondicionamento dos RQP, verificou-se no PGRSS que, o HU-USP adotou o coletor plástico para $\mathrm{RQ}$ em polietileno, rígido, resistente às perfurações em qualquer ponto de sua superfície, cor laranja, com tampa de rosca que assegura fechamento eficiente e simbologia de risco?.

No mesmo estudo, a autora relatou que no abrigo externo dos RQP, o coletor laranja é predominantemente utilizado para acondicionar frascos de medicamentos; como antibióticos, além de frascos, seringas, agulhas e demais materiais derivados da assistência aos pacientes tratados com antineoplásicos; observando ainda que resíduos de formaldeído e ortoftaldeído eram acondicionados na embalagem original do produto ${ }^{6}$.

Quanto ao volume de resíduos, há uma série de dados relevantes relacionados ao gerenciamento dos RSS hospitalares, com destaque à quantidade dos resíduos gerados por meio de pesagem antes da eliminação ${ }^{20}$.

No PGRSS, da mesma instituição deste estudo, verificou-se o registro da segregação dos RQP no período de 2001 a 2006. Ainda no referido PGRSS, evidenciou-se que os setores assistenciais como são os locais geradores dos RQP manuseados pela Enfermagem, além do serviço de Farmácia, serviço de anatomia patológica, laboratório clínico, hemocentro, manutenção, serviço de higiene especializada e audiometria ${ }^{7}$.

A segregação do formaldeído e do ortoftaldeído (substituto do glutaraldeído) gerados no centro obstétrico e serviço de endoscopia do HU-USP é confirmada nesta pesquisa.

Pesquisas evidenciaram que a quantidade produzida de RSS, inclusive dos RQP, varia, conforme o tipo de hospital e o tipo de assistência. O conhecimento das quantidades reais de resíduos gerados é de complicada determinação, sendo que raras vezes é obtido. Então, são usados valores estimados, já que, quanto mais complexo o serviço, maior será a possibilidade de produção de grandes volumes de RSS ${ }^{7,21-23}$.

O PGRSS deve ser elaborado, baseado nas características e no volume dos resíduos, como importantes indicadores no gerenciamento dos resíduos perigosos, inclusive para os RQP.

Quanto às identificações gerais dos recipientes de resíduos, durante as 16 avaliações, das 11(100\%) especificações da referida etapa, somente 2 (18,18\%) atenderam às recomendações da FIGERQP-HOSP, sendo representados pelas informações nome do hospital e endereço nos referidos recipientes, segundo expõe a tabela 2.

No que diz respeito à identificação dos RSS, destaca-se o fato de que a mesma deve estar presente nos recipientes de coleta interna e externa, nos sacos de acondicionamento, nos recipientes de transporte interno e externo, e nos locais de armazenamento².

A caracterização dos coletores, por meio de cores e identificação por símbolos, é uma prática mundial, com predomínio do acondicionamento de tecidos humanos em recipientes vermelhos, dos infectantes, e o grupo dos perfurocortantes em recipientes amarelos, e os demais em recipientes laranja ${ }^{24,25}$.

No Brasil, os resíduos infectantes são acondicionados em saco branco leitoso, os perfurocortantes em caixas amarelas e os químicos em coletores laranja, todos com suas respectivas simbologias de riscos ${ }^{7}$.

Para identificação do resíduo, a instituição campo deste estudo possui um impresso denominado Identificação do Resíduo com informações quanto ao tipo de resíduo, dados da instituição geradora, destinatário e simbologia.

Os RQ devem ser atentamente identificados no recipiente quanto à rotulagem correspondente ao nome do produto, propriedades físicas e químicas, volume, data de embalagem, horário, tipo de veículo de transporte, além do símbolo de perigo, que identifique as propriedades de periculosidade $\mathrm{e}^{6,21,23}$.

Neste estudo, verifica-se que as subinformações na identificação do recipiente quanto aos dados gerais da instituição, e sobre as informações relativas ao transporte interno, impactam, principalmente, no manejo intraestabelecimento com reflexo posterior no extraestabelecimento.

Ressalta-se também a necessidade de a instituição atentar para a Norma P4.262 da CETESB, a CVS 21 do Centro de Vigilância Sanitária e a NBR 14725-2 da Associação Brasileira de Normas Técnicas (ABNT) $)^{7,24,26}$.

Este estudo evidenciou que a falta de informações, no recipiente coletor, quanto à segregação e acondicionamento, pode comprometer as demais etapas de manejo, com destaque ao tratamento e disposição final.

TABELA 2: Especificações de identificações gerais em relação ao número total de avaliações nos recipientes de resíduos de formaldeído e ortoftaldeído. Brasil, 2012. ( $\mathrm{N}=16)$

\begin{tabular}{lcccc}
\hline $\begin{array}{l}\text { Especificações da etapa de } \\
\text { identificação geral no coletor }\end{array}$ & $\begin{array}{c}\text { Pre- } \\
\text { sente }\end{array}$ & \% & $\begin{array}{c}\text { Au- } \\
\text { sente }\end{array}$ & \% \\
\hline Nome do hospital & 16 & 100 & - & - \\
Endereço & 16 & 100 & - & - \\
No da embalagem & - & - & 16 & 100 \\
Enfermeiro do setor & - & - & 16 & 100 \\
Nome do responsável pelo & - & - & 16 & 100 \\
preenchimento & - & - & 16 & 100 \\
Nome do setor de origem & - & - & 16 & 100 \\
Nome do resíduo & - & - & 16 & 100 \\
$\begin{array}{l}\text { Quantidade do resíduo } \\
\text { Data de início de }\end{array}$ & - & & 16 & 100 \\
armazenamento & - & - & 16 & 100 \\
Identificação de 3/4 de & - & - & & \\
preenchimento do recipiente & - & & &
\end{tabular}


Na etapa de transporte interno, durantes as 16 avaliações, das 4 (100\%) especificações - data, horário, tipo de carimbo coletor e identificação do RQP no carrinho coletor - nenhum estava presente.

Para o transporte interno, a RDC № 306/2004 da ANVISA, definiu o transporte interno como o translado do resíduo desde seu local de geração até o abrigo interno ou externo ${ }^{2}$.

Dificuldades relativas aos recursos humanos e físicos fazem com que o transporte interno de RSS ocorra muitas vezes pela mão ou mediante o uso inadequado do carrinho transportador ${ }^{16}$.

A etapa de transporte interno deve ser representada em planta baixa, anexa ao PGRSS, favorecendo a identificação dos locais de geração de resíduos por grupo, seus respectivos fluxos, da geração até o abrigo externo.

Além da ausência de especificações de registros nos coletores e carrinhos, verificou-se ainda que a planta baixa do transporte interno não foi identificada no PGRSS do HU-USP. Dessa forma, a análise detalhada da adequação ou não do fluxo de transporte dos RQP derivados dos produtos identificados, não foi realizada.

A utilização de planta baixa, simbologia e legendas de cores colaboram no traçado do fluxo dos resíduos, facilitando as propostas para gerenciar os RSS e subsidiar o PGRSS 21

A aplicação da FIGERQP-HOSP na instituição deste estudo, possibilitou avaliação das especificações necessárias ao gerenciamento intra-hospitalar do formaldeído e ortoftaldeído, manuseados pela equipe de Enfermagem do Centro Obstétrico e Serviço de Endoscopia.

\section{CONCLUSÃo}

Neste estudo, verificou-se, por meio da FIGERQP-HOSP, que o GRQP de formaldeído e ortoftaldeído mostra uma série de não conformidades no atendimento às especificações de gerenciamento da segregação, acondicionamento, identificação e transporte interno dos resíduos de formaldeído e ortoftaldeído. Tai irregularidades podem acarretar diversos problemas, entre eles afetar a saúde dos trabalhadores de enfermagem, assim como o meio ambiente.

No entanto, vale destacar que importantes especificações do manejo dos $R Q$ vêm sendo atendidas, pela instituição campo de estudo. Ressalta-se o potencial da Comissão de Gerenciamento de Resíduos de Serviços de Saúde do HU-USP, em parceria com a administração da instituição e os órgãos públicos, para a realização dos ajustes necessários ao cumprimento das demais especificações, para o aprimoramento do sistema de rotulagem dos coletores dos RQP, e na adequação do Plano de Gerenciamento de Resíduos Químicos Perigosos, como parte integrante do PGRSS.

\section{REFERÊNCIAS}

1. Carvalho EC, David HMSL. A aplicação da precaução e da prevenção no ambiente de trabalho. Rev. enferm. UERJ. 2016; 24(3):e26180.

2. Ministério da Saúde (Br), Agência Nacional de Vigilância Sanitária. Resolução da Diretoria Colegiada - RDC no 306, de 07 de dezembro de 2004. Dispõe sobre o Regulamento Técnico para o gerenciamento de resíduos de serviços de saúde. Brasília (DF): ANVISA; 2004

3. Governo Estadual (SP), Companhia Ambiental do Estado de São Paulo. Informações toxicológicas. FIT-Ficha de Informação Toxicológica [site de Internet]. São Paulo: CETESB; 2013 [citado em 28 ago 2017]. Disponível em: https://cetesb.sp.gov.br/laboratorios/servicos/informacoes-toxicologicas/

4. Coggon D, Harris EC, Poole J, Palmer KT. Extended follow-up of a cohort of british chemical workers exposed to formaldehyde. J. Natl. Cancer Inst. 2003; 95(21): 1608-15.

5. Felli VEA, Baptista PCP. Saúde do trabalhador de enfermagem. São Paulo: Manole; 2015.

6. Psaltikidis EM, Leichsenring ML, Nakamura MHY, Bustorff-Silva JM, Passeri LA, Venâncio SI. Desinfetantes de alto nível alternativos ao glutaraldeído para processamento de endoscópios flexíveis. Cogitare enferm. Rev. 2014; 19(3):465-74.

7. Costa TF, Felli VEA, Baptista PCP. A percepção dos trabalhadores de enfermagem sobre o manejo dos resíduos químicos perigosos. Rev. Esc. Enferm. USP. 2012; 46(6):1453-61.

8. São Paulo (Estado), Companhia Ambiental do Estado de São Paulo. Norma técnica P4.262, de agosto de 2007. Dispõe sobre o gerenciamento de resíduos químicos provenientes de estabelecimentos de serviços de saúde [site de Internet]. São Paulo: CETESB; 2007 [citado em 30 out 2017]. Disponível em: https://www.cetesb. sp.gov.br/wp-content/uploads/2013/11/DD_224_DO.pdf

9. Ministério do Trabalho e Emprego (Br). Norma Regulamentadora $n$ ㅇ 32, de 11 de novembro de 2005. Dispõe sobre a segurança e saúde no trabalho em serviços de saúde. Brasília: Gabinete Ministerial; 2005.

10. BRasil (Br). Lei no. 12.305 de 2 de agosto de 2010. Institui a Política Nacional de Resíduos Sólidos; altera a Lei no. 9.605, de 12 de fevereiro de 1998; e dá outras providências. Diário Oficial da União [site de Internet]. 3 ago 2010 [citado em 05 ago 2017]; Seção 1. p. 3. Disponível em: http://www.planalto.gov.br/ccivil_03/_Ato2007-2010/2010/Lei/L12305.htm

11. Felli VEA, Costa TF, Baptista PCP, Guimarães ALO, Anginoni BM. Exposição dos trabalhadores de enfermagem às cargas de trabalho e suas consequências Rev. Esc. Enferm. USP. 2015; 49(esp 2):98-105.

12. Bdour A, Altrabsheh B, Hadadin N, Al-Shareif M. Assessment of medical wastes management practice: a case study of the northern part of Jordan. Waste Manag. 2007; 27(6):746-59.

13. Oweis R, Al-Widyan M, Al-Limoon O. Medical waste management in Jordan: a study at the King Hussein Medical Center. Waste Manag. 2005; 25(6):622-5.

14. Ferreira V, Teixeira MR. Healthcare waste management practices and risk perceptions: findings from hospitals in the Algarve region, Portugal. Waste Manag. 2010; 30(12):2657-63.

15. Tudor TL, Woolridge AC, Phillips CA, Holliday M, Laird K, Bannister $\mathrm{S}$, et al. Evaluating the link between the management of clinical waste in the National Health Service (NHS) and the risk of the spread of infections: a case study of three hospitals in England. Int. J. Hygiene Environ. Health. 2010; 213(6):432-6.

16.Soysal A, Simsek H, Soysal D, Alyu F. Management of health-care waste in Izmir, Turkey. Ann. Ist. Super Sanita. 2010; 46(3):299-302.

17. Tsakona M, Anagnostopoulou E, Gidarakos E. Hospital waste management and toxicity evaluation: a case study. Waste Manag. 2007; 27(7):912-20. 
18. Costa AMP. Elaboração e avaliação da implantação de um modelo básico de plano de gerenciamento de resíduos de serviços de saúde em unidades hospitalares da Região Metropolitana da Baixada Santista [dissertação de mestrado]. São Paulo: Universidade de São Paulo; 2001.

19. Garcia LP, Zanetti-Ramos BG. Gerenciamento dos resíduos de serviços de saúde: uma questão de biossegurança. Cad. Saúde Pública (Online). 2004;20(3):744-52.

20.Jang YC, Lee C, Yoon OS, Kim H. Medical waste management in Korea. J. Environ. Manage. 2006; 80(2):107-15.

21. Bendjoudi Z, Taleb F, Abdelmalek F, Addou A. Healthcare waste management in Algeria and Mostaganem department. Waste Manag. 2009; 29(4):1383-7.

22. Sales JEY, Silva EB. Guia de descarte de produtos químicos perigosos de laboratório. São Paulo: UNESP; 2016.
23. Takayanagui AMM. Risco ambiental e o gerenciamento de resíduos nos espaços de um serviço no Canadá: um estudo de caso [tese de livre docência]. Ribeirão Preto (SP): Universidade de São Paulo; 2004. 24. Secretaria de Estado da Saúde (SP). Centro de Vigilância Sanitária. Portaria CVS no 21, de 10 de setembro de 2008. Aprova a Norma Técnica sobre Gerenciamento de Resíduos Perigosos de Medicamentos em Serviços de Saúde. São Paulo: Centro de Vigilância Sanitária; 2008.

25. Oliveira LL, Souza PM, Clementino FS, Paiva SC, Rocha FDLJ. Resíduos dos serviços de saúde: desafios e perspectivas na atenção primária. Rev. enferm. UERJ. 2014;22(1):29-34.

26. Associação Brasileira de Normas Técnicas. NBR 14725-2 Produtos químicos: informações sobre segurança, saúde e meio ambiente: parte 2: sistema de classificação de perigo. Rio de Janeiro: ABNT; 2009. 